\title{
ERRATUM
}

\section{Spin injection/detection using an organic-based magnetic semiconductor}

Jung-Woo Yoo, Chia-Yi Chen, H. W. Jang, C. W. Bark, V. N. Prigodin, C. B. Eom \& A. J. Epstein

Nature Materials 9, 638-642 (2010); published online: 18 July 2010; corrected after print: 23 July 2010.

In the original version of this article published online and in print, the received date should have been 29 December 2009, not 2010. This has been corrected in the PDF and HTML versions of this Letter. 